\title{
HMGA1 correlates with advanced tumor grade and decreased survival in pancreatic ductal adenocarcinoma
}

\author{
Alexandra C Hristov ${ }^{1}$, Leslie Cope ${ }^{2,3}$, Francescopaolo Di Cello ${ }^{4}$, Marcelo Delos Reyes ${ }^{1}$, \\ Mansher Singh ${ }^{1}$, Joelle A Hillion ${ }^{4}$, Amy Belton ${ }^{4}$, Biju Joseph ${ }^{4}$, Andrew Schuldenfrei ${ }^{4,5}$, \\ Christine A Iacobuzio-Donahue ${ }^{1,2}$, Anirban Maitra ${ }^{1,2}$ and Linda MS Resar ${ }^{2,4,5}$ \\ ${ }^{1}$ Department of Pathology, the Johns Hopkins University School of Medicine, Baltimore, MD, USA; \\ ${ }^{2}$ Department of Oncology, the Johns Hopkins University School of Medicine, Baltimore, MD, USA; \\ ${ }^{3}$ Department of Biostatistics, the Johns Hopkins University School of Medicine, Baltimore, MD, USA; \\ ${ }^{4}$ Department of Medicine, the Johns Hopkins University School of Medicine, Baltimore, MD, USA and \\ ${ }^{5}$ Department of Pediatrics, the Johns Hopkins University School of Medicine, Baltimore, MD, USA
}

\begin{abstract}
Although pancreatic ductal adenocarcinoma is a common and almost uniformly fatal cancer, little is known about the molecular events that lead to tumor progression. The high-mobility group A1 (HMGA1) protein is an architectural transcription factor that has been implicated in the pathogenesis and progression of diverse human cancers, including pancreatic ductal adenocarcinoma. In this study, we investigated HMGA1 expression in pancreatic ductal adenocarcinoma cell lines and surgically resected tumors to determine whether it could be a marker for more advanced disease. By real-time quantitative RT-PCR, we measured HMGA1a mRNA in cultured pancreatic ductal adenocarcinoma cell lines and found increased levels in all cancer cells compared with normal pancreatic tissue. To investigate HMGA1 in primary human tumors, we performed immunohistochemical analysis of 125 cases of pancreatic adenocarcinoma and 99 precursor lesions (PanIN 1-3). We found nuclear staining for HMGA1 in $98 \%$ of cases of pancreatic adenocarcinoma, but only $43 \%$ of cases of PanIN precursor lesions. Moreover, HMGA1 immunoreactivity correlates positively with decreased survival and advanced tumor and PanIN grade. These results suggest that HMGA1 promotes tumor progression in pancreatic ductal adenocarcinoma and could be a useful biomarker and rational therapeutic target in advanced disease. Modern Pathology (2010) 23, 98-104; doi:10.1038/modpathol.2009.139; published online 9 October 2009
\end{abstract}

Keywords: HMGA1; pancreatic ductal adenocarcinoma; oncogene; immunoreactivity

Although pancreatic ductal adenocarcinoma is common and highly lethal, little progress has been made in the treatment of this disease. ${ }^{1}$ In the United States, more than 34000 patients succumb to pancreatic ductal adenocarcinoma annually, making this malignancy the fourth leading cause of cancer death. ${ }^{1}$ Most patients present with locally invasive or metastatic disease, for which there are no effective therapies. Even in patients with small primary lesions that are amenable to surgical resection, most will eventually die from metastatic progression despite adjuvant chemotherapy and radiation ther-

Correspondence: Dr LMS Resar, The Johns Hopkins University School of Medicine, Ross Research Building, Room 1025, 720 Rutland Ave, Baltimore, MD 21205, USA.

E-mail: lresar@jhmi.edu

Received 20 April 2009; revised 8 July 2009; accepted 21 July 2009; published online 9 October 2009 apy. ${ }^{1,2}$ These dismal epidemiological data suggest that pancreatic cancers metastasize before clinical detection in the majority of cases. Moreover, the abysmal outcome for most patients with pancreatic ductal adenocarcinoma underscores the urgent need to discover molecular pathways involved in tumor progression to facilitate the development of more effective therapies. Although progress has been made in elucidating early molecular lesions that lead to tumor initiation, little is known about those that underlie tumor progression and eventual metastases.

Pancreatic ductal adenocarcinomas are caused by noninvasive, precursor lesions after a series of wellcharacterized, stepwise molecular alterations that correspond to distinct histopathological entities. ${ }^{2-11}$ These precursor lesions, termed 'pancreatic intraepithelial neoplasia (PanIN),' are divided into three grades using an international classification system 
that is based on histopathological features, including the degree of architectural and cytological atypia. ${ }^{3-6}$ PanIN-1, a relatively common lesion, can be identified in approximately a third of all elderly persons, but epidemiological data suggests that it rarely becomes malignant. ${ }^{3-6}$ PanIN-1 lesions are further divided into PanIN-1A and PanIN-1B lesions on the basis of the presence of a papillary, micropapillary or a basally pseudostratified architecture identified only in PanIN-1B. ${ }^{4-6}$ Activation of the K-RAS oncogene and telomere shortening have been identified in PanIN-1 lesions, suggesting that these lesions could be early in tumor initiation. ${ }^{4-8}$ The intermediate stage, PanIN-2, is far more likely to be found in pancreata harboring invasive cancer. ${ }^{8}$ Loss of function of p16 (INK4A) by deletion, methylation, or mutation is a common molecular alteration present in PanIN-2 lesions. ${ }^{9}$ PanIN-3, formerly known as 'carcinoma-in-situ,' immediately precedes the development of invasive carcinoma, and is often found in association with adjacent invasive cancer. $^{3-11}$ Mutations that alter TP53 and MAD4/DPC4 are frequently found in PanIN-3 lesions that are present in association with sporadic pancreatic cancer. ${ }^{10,11}$ Studies to elucidate molecular lesions that drive the transformation of PanIN-3 to invasive carcinoma are lacking.

The high mobility group A (HMGA1) oncogene is widely expressed during embryonic development, ${ }^{12}$ and is overexpressed in diverse, high-grade human cancers, including pancreatic ductal adenocarcinoma. ${ }^{13-26}$ This gene encodes the HMGA1a and HMGA1b protein isoforms, which function in modulating gene expression. ${ }^{13-25}$ Increasing evidence indicates an important role for these proteins in promoting tumor progression. ${ }^{13-24}$ HMGA1 proteins induce a transformed phenotype with anchorage-independent cell growth in immortalized, cultured cells derived from diverse normal tissue. ${ }^{14,15,17,18,27}$ Furthermore, forced overexpression of HMGA1 is associated with resistance to anoikis in pancreatic ductal adenocarcinoma cell lines. ${ }^{22}$ Interfering with its expression inhibits anchorage-independent cell growth, cellular motility, migration, and invasion in some cancer cell lines. ${ }^{14,15,17-22,26}$ In addition, transgenic mice overexpressing HMGA1 develop aggressive malignancies. ${ }^{19,21,25}$ More recently, studies with an orthotopic tumor mouse model showed that knockdown of HMGA1 expression in human pancreatic cancer cells blocked tumor growth and metastatic progression. ${ }^{20,26}$ Taken together, these findings suggest that HMGA1 could participate in transformation in pancreatic ductal adenocarcinoma and promote tumor progression.

In this study, we investigated HMGA1 expression at the mRNA and protein levels in pancreatic ductal adenocarcinoma. We found that HMGA1 is overexpressed in cultured pancreatic ductal adenocarcinoma cell lines compared with normal pancreatic tissue. Moreover, increasing protein levels correlate with decreased survival and more advanced tumor and PanIN grade. Although further studies are required, our findings suggest that HMGA1 may be an important driver of tumor progession and potential therapeutic target in this highly lethal malignancy.

\section{Materials and methods}

\section{Cultured Human Pancreatic Ductal Adenocarcinoma Cells and Normal Human Pancreas}

The pancreatic ductal adenocarcinoma cell lines have been described previously. ${ }^{28}$ Briefly, MIA PaCa-2 (CRL-1420), and PL-1 (Panc 02.13, CRL2554) were obtained from the American Type Culture Collection and were cultured as recommended. MIA PaCa-2 cells were generated from a primary undifferentiated pancreatic carcinoma in a 65-yearold Caucasion man that involved the pancreatic body and tail and extended to involve the periaortic soft tissues. ${ }^{29} \mathrm{PL}-1$ cells were derived from a primary pancreatic ductal adenocarcinoma in a 64-year-old Caucasian woman that involved the duodenum, but did not metastasize to lymph nodes. ${ }^{30}$ XPA-3 (PX154) cells are xenografted tumor cells and were kindly provided by Dr Anirban Maitra. ${ }^{31}$ They originate from a primary pancreatic ductal adenocarcinoma in a 77-year-old man that extended to involve the duodenum and peripancreatic soft tissues and metastasized to lymph nodes. Normal human pancreas was obtained from a 35-year-old man (Becton Dickinson, San Jose, CA, USA).

\section{Quantitative RT-PCR of Cultured Human Pancreatic Ductal Adenocarcinoma Cells and Normal Human Pancreas}

Quantitative RT-PCR was performed by Taqman chemistry according to our previous report. ${ }^{19} H M G A 1 a$ mRNA levels were normalized to human phosphoprotein as an internal control. Repeat experiments with normalization to the additional control genes, $\beta$-glucuronidase and $\beta$-actin, were also conducted.

\section{Tissue Microarrays}

Tissue samples were obtained from the pathology archives of The Johns Hopkins Medical Institutions (JHMI, Baltimore, MD, USA) as described previously. ${ }^{28}$ Briefly, four 2-mm cores of pancreatic ductal adenocarcinoma were obtained from 125 patients, including two cores obtained from tumor in the pancreatic parenchyma and two cores obtained from tumor in the peripancreatic soft tissue and lymph nodes when infiltrated. Pathological diagnosis, grade and stage, as well as patient survival information were retrieved from the medical record. Staging was determined according to the American Joint Committee on Cancer guidelines. ${ }^{32}$ PanIN tissue microarray blocks were also created as described previously. ${ }^{33}$ In short, 90 PanIN lesions were selected from 33 patients with pancreatic 
ductal adenocarcinoma. Nine additional PanIN lesions from eight separate cases were identified on the pancreatic ductal adenocarcinoma tissue microarrays. PanIN lesions were separated into PanIN-1, PanIN-2 or PanIN-3 grades either at the time of microarray creation or after a review by AH.

\section{Immunohistochemistry}

Immunohistochemistry was performed as we described previously. ${ }^{28}$ Each pancreatic ductal adenocarcinoma tissue microarray was immunohistochemically stained for HMGA1 protein (HMGA1, dilution 1:25 from a stock solution of $200 \mu \mathrm{g} / \mathrm{ml}$, Santa Cruz Biotechnologies, no. sc-8982, Santa Cruz, CA, USA) following the manufacturer directions. Specific nuclear immunoreactivity of more than 5\% of neoplastic cells was interpreted as positive HMGA1 staining and was scored using a 9-point scale on the basis of the product of staining intensity (no staining $=0$, weak $=1$, moderate $=2$, strong $=3$ ) and staining extent ( $\%$ of positive cells; $<5 \%=0$, $5-30 \%=1,30-60 \%=2,>60 \%=3)$. The highest score per case was used for subsequent analyses. PanIN tissue microarrays were also stained for HMGA1 and interpreted as described above. All scores for PanIN lesions were used for subsequent analysis. At the dilutions used in this study, there was no significant cross-reactivity with HMGA2 using an antibody shown to be specific for HMGA2. ${ }^{28}$ The tissue microarrays were evaluated microscopically in a blinded manner by a single investigator (AH).

\section{Statistical Analysis}

Results are expressed as mean \pm s.e. Student's $t$-test was used to compare HMGA1 mRNA levels from pancreatic ductal adenocarcinoma cells and normal tissues. To determine whether HMGA1 immunoreactivity correlated with more advanced disease, clinical and pathological parameters were investigated, including overall survival, tumor grade, lymph node metastases and stage. A nonparametric rank-sum test was used to evaluate the relationship between HMGA1 immunoreactivity and survival. HMGA1 protein levels were compared with tumor grade, lymph node metastases, and stage using the $\chi^{2}$ test. Furthermore, HMGA1 protein expression was compared with PanIN grade using the two-sided Fisher's exact test.

\section{Results}

\section{HMGA1a Overexpression in Cultured Pancreatic Ductal Adenocarcinoma Cells}

To determine whether HMGA1a is overexpressed in pancreatic ductal adenocarcinoma, we measured HMGA1a mRNA levels by quantitative RT-PCR in cultured pancreatic ductal adenocarcinoma cells. We observed increased levels in all cultured cells

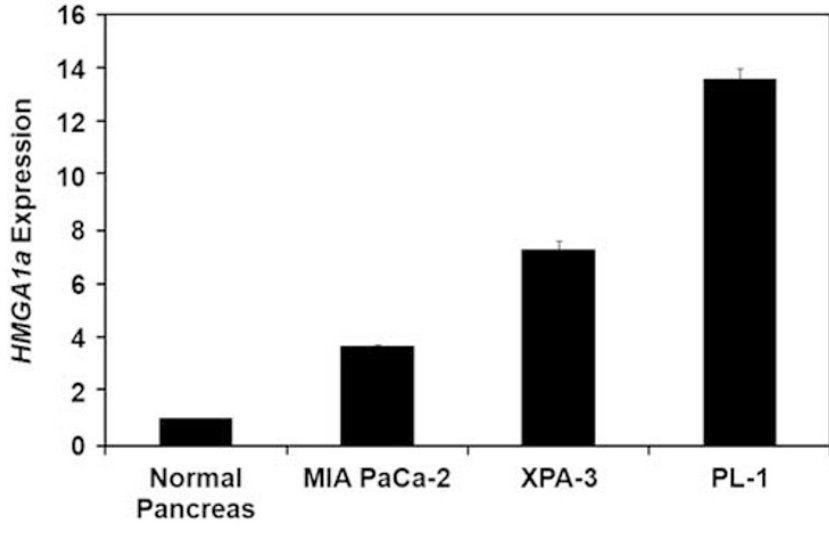

Figure 1 HMGA1a mRNA expression in human pancreatic ductal adenocarcinoma cell lines. HMGA1a mRNA levels were measured by quantitative RT-PCR and normalized to human phosphoprotein. Normal human pancreatic tissue from a healthy 35-year-old donor was used as a control and assigned a value of 1. HMGA1a mRNA levels are significantly above control in all cell lines (4-fold $\pm 0.08, P<0.0001$ for MIA PaCa-2 cells; 7 -fold \pm 0.3 , $P<0.0001$ for XPA-3 cells and 14 -fold $\pm 0.4, P<0.0001$ for PL- 1 cells vs $1 \pm 0.05$ for normal human pancreas). Three independent experiments were performed in triplicate; results represent the mean \pm s.e. from independent experiments. Normalization to $\beta$-glucuronidase and $\beta$-actin as control genes produced similar results in separate experiments (not shown).

Table 1 Distribution of cases represented on the pancreatic ductal adenocarcinoma tissue microarrays by stage, lymph node involvement, grade and mean survival

Grade Survival (months)

\begin{tabular}{lrrrrrrr}
\cline { 3 - 6 } Stage & $\mathrm{n}$ & $N 1^{\mathrm{a}}$ & 1 & 2 & 3 & Unknown & \\
\hline Ia/b & 3 & 0 & 0 & 2 & 1 & & 31.0 \\
IIa & 18 & 0 & 2 & 10 & 6 & & 18.4 \\
IIb & 101 & 101 & 2 & 53 & 43 & 3 & 17.4 \\
III & 3 & 2 & 0 & 1 & 2 & & 13.3 \\
\hline
\end{tabular}

${ }^{\mathrm{a}} \mathrm{N} 1$ indicates lymph node involvement.

compared with HMGA1a mRNA levels in normal pancreatic tissue (Figure 1). Notably, HMGA1 mRNA was increased by fourfold in MIA PaCa-2 cells $(P<$ 0.001 by Student's $t$-test), sevenfold in the XPA-3 $(P<0.001$ by Student's $t$-test), and 14 -fold in PL-1 cells $(P<0.001$ by Student's t-test). All of these cell lines were derived from tumors from patients with advanced disease. MIA PaCa-2 cells originate from an undifferentiated pancreatic carcinoma that extended to periaortic tissues. ${ }^{29}$ XPA-3 cells were generated from a primary tumor that extensively involved regional tissues and metastasized to lymph nodes $^{31}$ and PL-1 cells are from a primary tumor that extended to the duodenum. ${ }^{30}$

\section{Patient Characteristics}

The mean age for patients was 67.5 years (range: $32-$ 90 years). 64 patients were men and 61 patients were women. Table 1 displays the available stage, grade 

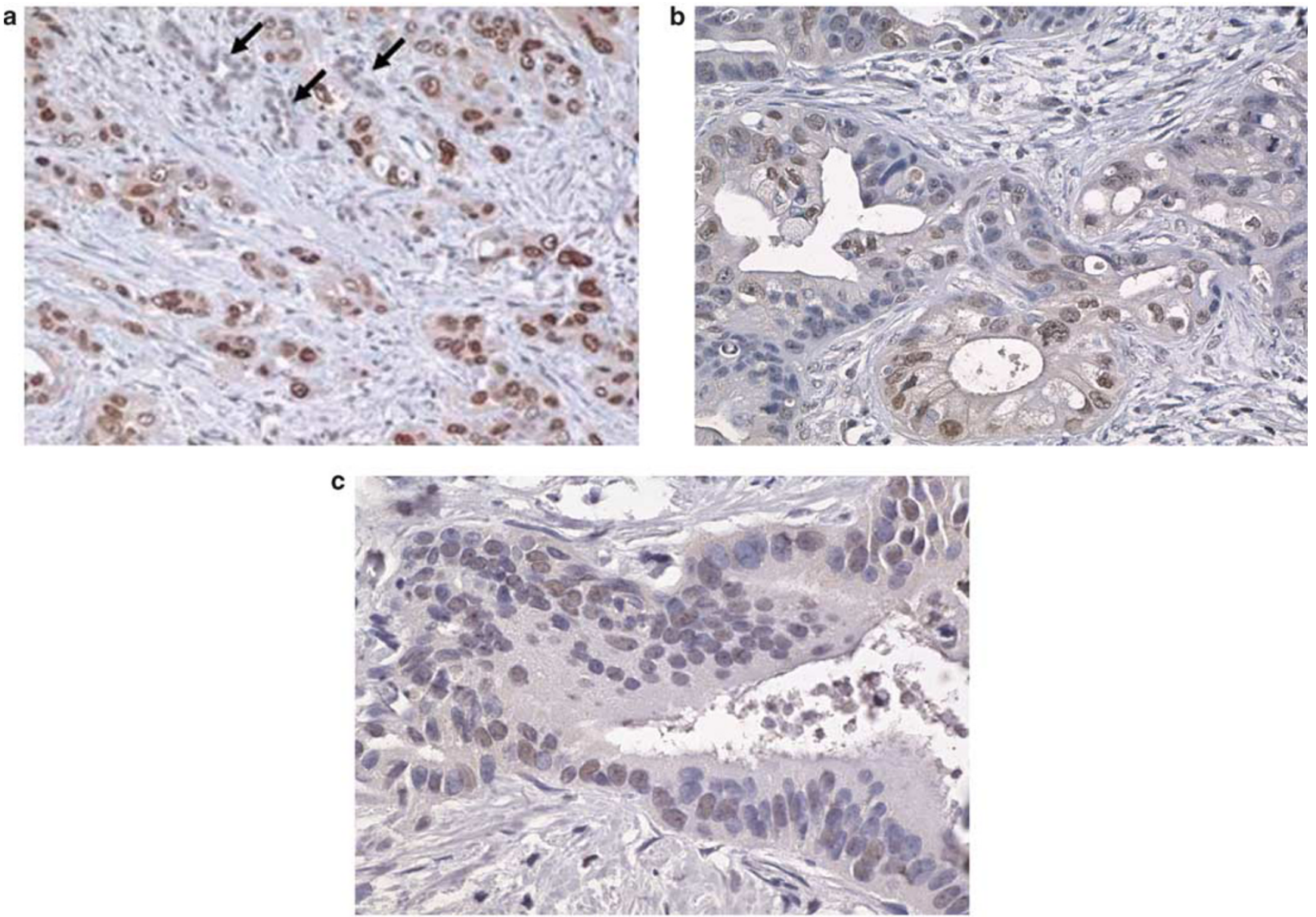

Figure 2 HMGA1 protein was detected in the majority of PDA by immunohistochemistry and increased levels of HMGA1 immunoreactivity correlated inversely with tumor differentiation (HMGA1 antibody, Gill hematoxylin counterstain). (a) Strong immunoreactivity in poorly-differentiated pancreatic ductal adenocarcinoma (magnification X 64, score 9). There is limited HMGA1 reactivity in benign pancreatic ducts (arrows). (b) Moderate immunoreactivity in moderately-differentiated pancreatic ductal adenocarcinoma (magnification X 100, score 6). (c) Weak immunoreactivity in well-differentiated pancreatic ductal adenocarcinoma (magnification X 160, score 2).

and survival information for these patients. Tumor grade was available for 122 of 125 patients. Survival information was available for 120 of 125 patients. Clinical observation ranged from 2 to 47 months $(23.4 \pm 9.8$, mean \pm s.d. $)$, and 67 patients expired during this study period.

\section{HMGA1 Immunoreactivity in Pancreatic Ductal Adenocarcinoma}

As HMGA1a was overexpressed in all pancreatic ductal adenocarcinoma cell lines studied, we next sought to determine whether HMGA1 protein is overexpressed in more advanced disease in pancreatic ductal adenocarcinoma. To this end, we assessed HMGA1 protein levels by immunohistochemical analysis in tissue microarrays that included normal pancreatic tissue, PanIN 1-3 lesions, and primary pancreatic ductal adenocarcinomas from tumors with varied grades of differentiation (well-differentiated $(n=4)$, moderately differen- tiated $(n=66)$, poorly differentiated $(n=52))$. HMGA1 immunoreactivity was identified in 123 of $125(98 \%)$ cases of pancreatic ductal adenocarcinoma, with scores ranging from 1 to 9 (Figure 2). Of positive cases, $22(18 \%)$ displayed limited reactivity (score <3), $53(42 \%)$ displayed moderate reactivity (score 3-6) and $48(39 \%)$ displayed strong reactivity (score >6). Normal tissues represented on the tissue microarrays, including benign pancreatic ducts, displayed limited to no HMGA1 immunoreactivity. Benign biliary epithelium was a notable exception and occasionally displayed moderate-tostrong HMGA1 nuclear immunoreactivity in the majority of cells.

\section{HMGA1 Protein Expression Correlates with Patient Survival and Tumor Grade}

As HMGA1 immunoreactivity was increased in the pancreatic adenocarcinoma samples compared with normal pancreatic tissue, we hypothesized that it 


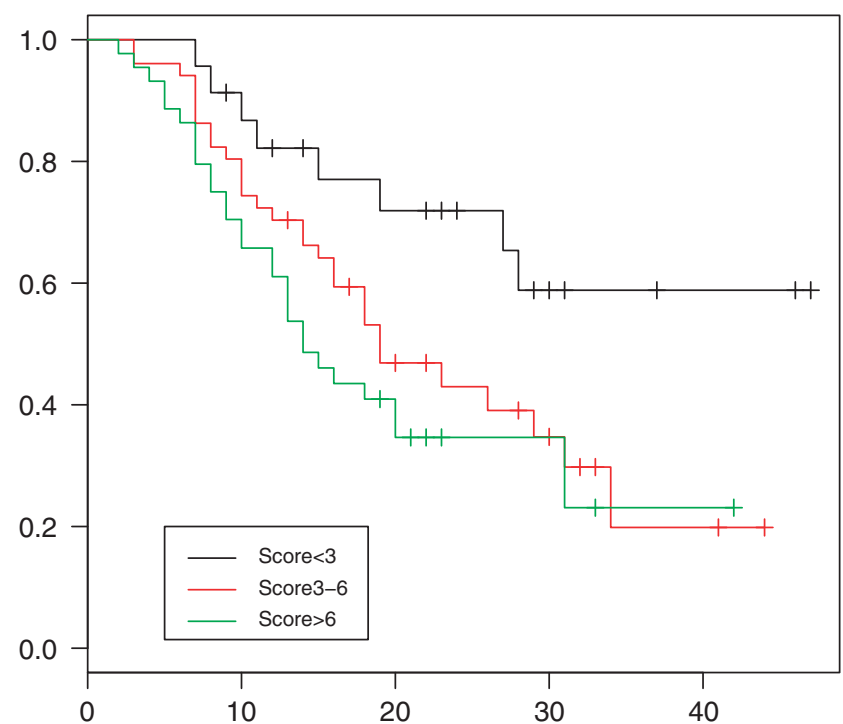

Figure 3 A Kaplan-Meier curve demonstrates that HMGA1 reactivity inversely related to mean survival. Patients with limited to no HMGA1 reactivity (score $<3$ ) had longer survival than those with moderate or strong HMGA1 reactivity (score 3-6 and score $>6$, respectively). Further, patients with the strongest HMGA1 reactivity (score $>6$ ) had the shortest survival $(P=0.0175$ by nonparametric rank sum test $)$.

could be a marker for more advanced disease. We therefore determined whether HMGA1 protein levels correlate with clinicopathologic parameters indicative of disease progression, including decreased survival, poor tumor differentiation (high grade), lymph node metastases, and stage. We found that HMGA1 immunoreactivity was inversely associated with mean survival. Patients with limited to no reactivity had longer survival than those with strong reactivity $(<3$ : 23.9 months, 3-6: 17.6 months, $>6$ : 15.0 months, $P=0.0175$ by nonparametric rank-sum test, Figure 3). Furthermore, increased immunoreactivity for HMGA1 was positively correlated with tumor grade. Tumors with an HMGA1 score of $\geq 4$ were less differentiated when compared with tumors with an HMGA score $<4 \quad(P=0.03$, $\chi^{2}$ test). We did not find a correlation between HMGA1 immunoreactivity and lymph node metastases or overall stage.

\section{HMGA1 Protein Levels are Increased in High-Grade PanINs}

Only 43\% (43/99) of PanINs demonstrated nuclear HMGA1 immunoreactivity. Of these HMGA1-positive lesions, 86\% (37/43) displayed low HMGA1 protein expression (score <3). Notably, PanIN-3 lesions were the most likely to have demonstrable HMGA1 staining. In fact, HMGA1 staining was positively correlated with increasing PanIN grade. Specifically, $78 \%$ of PanIN-3 lesions and $55 \%$ of PanIN-2 lesions exhibited HMGA1 staining, whereas only $36 \%$ of PanIN-1 lesions exhibited HMGA1
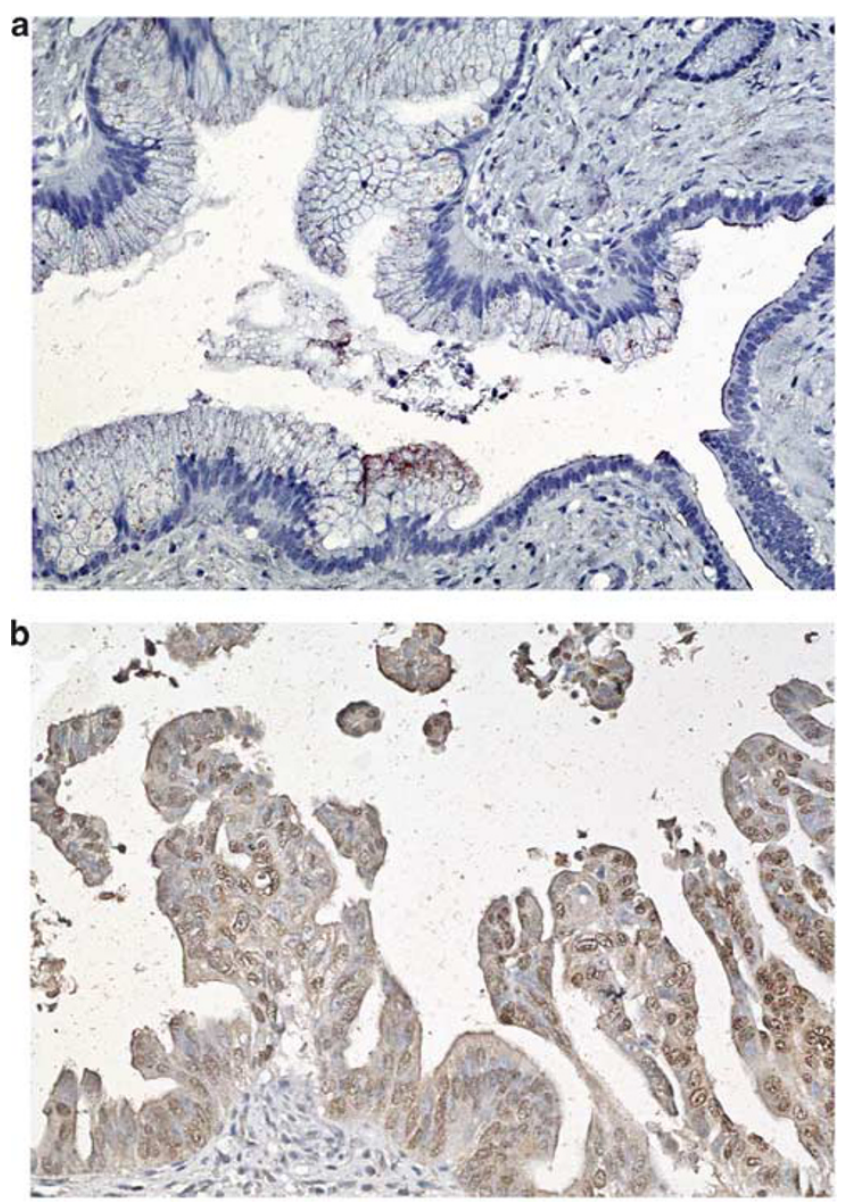

Figure 4 HMGA1 protein expression in PanIN determined by immunohistochemistry correlates with grade (HMGA1 antibody, Gill hematoxylin counterstain). (a) No HMGA1 immunoreactivity in a low grade PanIN lesion (PanIN-1B). (b) High HMGA1 immunoreactivity in a high grade PanIN lesion (PanIN-3).

immunoreactivity $(P=0.024$ by the two-sided Fisher's test, Figure 4).

\section{Discussion}

Recent studies have identified molecular alterations that occur in PanIN precursor lesions as they progress to invasive ductal adenocarcinoma. Activating mutations in the $K-R A S 2$ gene and telomere shortening are believed to occur early because they are often observed in PanIN-1 lesions. ${ }^{4-6}$ Mutations that inactivate $p 16(I N K 4 A)$ are frequently seen in PanIN-2 lesions, ${ }^{9}$ whereas inactivation of TP53 and MAD4/DPC4 are typically observed in PanIN-3 lesions. ${ }^{3,10,11}$ Unfortunately, elucidation of these early molecular events has not translated into improved patient survival. Moreover, the changes that contribute to tumor invasion, malignant progression, and eventual metastasis are poorly understood. As most patients with pancreatic cancer present with advanced disease or rapidly develop distant metastases, discoveries of later molecular 
events that cause tumor progression are required to identify cellular pathways that could be targeted in therapy.

Previous studies have implicated HMGA genes and proteins in the pathogenesis of pancreatic ductal adenocarcinoma and other high-grade tumors. We recently showed that another HMGA family member, HMGA2, is overexpressed in pancreatic ductal adenocarcinoma cell lines. ${ }^{28}$ Moreover, we found that HMGA2 protein levels correlate with lymph node metastases and tumor grade by immunohistochemical analysis of primary human tumors. In another study using immunohistochemical analysis, HMGA1 protein was found to be expressed in 15 ductal adenocarcinomas, including 6 metastatic lesions, but not in benign ductal epithelium. There was no correlation between protein levels and tumor differentiation in this relatively small, pilot study. ${ }^{16}$ HMGA1 protein was also found to be increased in two intraductal papillary mucinous neoplasms (IPMNs). ${ }^{16}$ In a subsequent paper, this group also demonstrated increased HMGA1 protein in pancreatic ductal adenocarcinoma cell lines using Western blot analysis, with no demonstrable levels in normal pancreas. $^{34}$ More recently, another group demonstrated HMGA1 immunoreactivity in $93 \%$ of pancreatic ductal adenocarcinomas from 89 patients. ${ }^{26}$ In this study, only the intensity of HMGA1 protein expression was determined, and statistical analyses were based on the presence or absence of immunoreactivity. Unlike our study, no correlation between HMGA1 staining and tumor differentiation was detected, although this could relate to the dichotomized analysis or the smaller sample size in the previous study. ${ }^{26}$ However, similar to our study, there was a significant correlation between HMGA1 immunoreactivity and survival. It is noteworthy that this previous study included a very limited number of samples without HMGA1 immunoreactivity ( $n=6$, or $7 \%$ ) as the basis for their survival analysis. In addition, it was not clear whether HMGA1 expression was an early or late event in tumor progression because PanIN lesions were not examined. Functional studies in pancreatic ductal adenocarcinoma cell lines showed that knockdown of HMGA1 interferes with cellular invasion, ${ }^{20}$ anchorage-independent cell growth, ${ }^{26}$ resistance to anoikis, ${ }^{22}$ and resistance to gemcitabine. ${ }^{35,36}$ Taken together, these findings indicate that $H M G A 1$ could drive tumor progression in pancreatic ductal adenocarcinoma.

In summary, we report that HMGA1 is overexpressed in pancreatic ductal adenocarcinoma. We found high levels of HMGA1 mRNA in pancreatic carcinoma cell lines compared with normal tissue. In addition, HMGA1 proteins are present in high levels in pancreatic ductal adenocarcinoma, but not in benign pancreatic ductal epithelium. Furthermore, we show that HMGA1 protein immunoreactivity correlates with measures of tumor progression, including advanced tumor grade and decreased survival. Because HMGA1 is not expressed in the majority of PanIN lesions and found predominantly in invasive carcinoma, our results suggest that it drives tumor progression. Together with functional studies demonstrating a role for HMGA1 in tumor invasion, anchorage-independent cell growth, and metastatic progression, these findings indicate that HMGA1 could also serve as a rational therapeutic target and potential biomarker in advanced disease. Future studies are required to identify critical downstream pathways induced by HMGA1 that are amenable to therapeutic interventions.

\section{Acknowledgements}

The authors kindly thank the generous support of the Joseph C. Eggleston Award and the Eggleston family, the American Cancer Society Scholar Award, Alex's Lemonade Stand Foundation, the J.P. McCarthy Fund, the Flight Attendant Medical Research Institute Young Clinical Investigator Award, the Prevent Cancer Foundation and the Maryland Stem Cell Research Fund. Also, the authors sincerely thank Dr. Ralph Hruban for his time and contributions to this study.

Grant support: American Cancer Society Scholar Award, R01 CA092339 and R03 CA139331-01 (L. M. S. Resar), Flight Attendant Medical Research Institute Young Clinical Investigator Award (F. Di Cello), Prevent Cancer Foundation (J. Hillion), Alex's Lemonade Stand Foundation (L.M.S. Resar and J. Hillion), Joseph C. Eggleston Award (A. Hristov), Maryland Stem Cell Research Fund (L.M.S. Resar, A. Belton, B. Joseph, and J. Hillion), J.P. McCarthy Fund (L.M.S. Resar), and NIH T32 grant (A. Belton).

\section{Disclosure/conflict of interest}

The authors declare no conflict of interest.

\section{References}

1 Jemal A, Siegel R, Ward E, et al. Cancer statistics, 2008. CA Cancer J Clin 2008;58:71-96.

2 Yeo TP, Hruban RH, Leach SD, et al. Pancreatic cancer. Curr Probl Cancer 2002;26:176-275.

3 Cubilla AL, Fitzgerald PJ. Morphological lesions associated with human primary invasive nonendocrine pancreas cancer. Cancer Res 1976;36:2690-2698.

4 Hruban RH, Adsay NV, Albores-Saavedra J, et al. Pancreatic intraepithelial neoplasia: a new nomenclature and classification system for pancreatic duct lesions. Am J Surg Pathol 2001;25:579-586.

5 Hruban RH, Maitra A, Goggins M. Update on pancreatic intraepithelial neoplasia. Int J Clin Exp Pathol 2008;1:306-316. 
6 Singh M, Maitra A. Precursor lesions of pancreatic cancer: molecular pathology and clinical implications. Pancreatology 2007;7:9-19.

7 van Heek NT, Meeker AK, Kern SE, et al. Telomere shortening is nearly universal in pancreatic intraepithelial neoplasia. Am J Pathol 2002;161:1541-1547.

8 Lohr M, Kloppel G, Maisonneuve P, Lowenfels AB, Luttges J. Frequency of K-ras mutations in pancreatic intraductal neoplasias associated with pancreatic ductal adenocarcinoma and chronic pancreatitis: a meta-analysis. Neoplasia 2005;7:17-23.

9 Wilentz RE, Geradts J, Maynard R, et al. Inactivation of the p16 (INK4A) tumor-suppressor gene in pancreatic duct lesions: loss of intranuclear expression. Cancer Res 1998;58:4740-4744.

10 Wilentz RE, Iacobuzio-Donahue CA, Argani P, et al. Loss of expression of Dpc4 in pancreatic intraepithelial neoplasia: evidence that DPC4 inactivation occurs late in neoplastic progression. Cancer Res 2000;60:2002-2006.

11 Luttges J, Galehdari H, Brocker V, et al. Allelic loss is often the first hit in the biallelic inactivation of the p53 and DPC4 genes during pancreatic carcinogenesis. Am J Pathol 2001;158:1677-1683.

12 Chiappetta G, Avantaggiato V, Visconti R, et al. High level expression of the HMGI (Y) gene during embryonic development. Oncogene 1996;13:2439-2446.

13 Fusco A, Fedele M. Roles of HMGA proteins in cancer. Nat Rev Cancer 2007;7:899-910.

14 Wood LJ, Maher JF, Bunton TE, Resar LM. The oncogenic properties of the HMG-I gene family. Cancer Res 2000;60:4256-4261.

15 Wood LJ, Mukherjee M, Dolde CE, et al. HMG-I/Y, a new c-Myc target gene and potential oncogene. Mol Cell Biol 2000;20:5490-5502.

16 Abe N, Watanabe T, Masaki T, et al. Pancreatic duct cell carcinomas express high levels of high mobility group I(Y) proteins. Cancer Res 2000;60:3117-3122.

17 Reeves R. Molecular biology of HMGA proteins: hubs of nuclear function. Gene 2001;277:63-81.

18 Dolde CE, Mukherjee M, Cho C, Resar LM. HMG-I/Y in human breast cancer cell lines. Breast Cancer Res Treat 2002;71:181-191.

$19 \mathrm{Xu}$ Y, Sumter TF, Bhattacharya R, et al. The HMG-I oncogene causes highly penetrant, aggressive lymphoid malignancy in transgenic mice and is overexpressed in human leukemia. Cancer Res 2004;64: 3371-3375.

20 Liau SS, Jazag A, Whang EE. HMGA1 is a determinant of cellular invasiveness and in vivo metastatic potential in pancreatic adenocarcinoma. Cancer Res 2006;66:11613-11622.

21 Tesfaye A, Di Cello F, Hillion J, et al. The high-mobility group A1 gene up-regulates cyclooxygenase 2 expression in uterine tumorigenesis. Cancer Res 2007;67:3998-4004.

22 Liau SS, Jazag A, Ito K, Whang EE. Overexpression of HMGA1 promotes anoikis resistance and constitutive
Akt activation in pancreatic adenocarcinoma cells. Br J Cancer 2007;96:993-1000.

23 Hillion J, Dhara S, Sumter TF, et al. The high-mobility group A1a/signal transducer and activator of transcription-3 axis: an achilles heel for hematopoietic malignancies? Cancer Res 2008;68:10121-10127.

24 Sarhadi VK, Wikman H, Salmenkivi K, et al. Increased expression of high mobility group A proteins in lung cancer. J Pathol 2006;209:206-212.

25 Fedele M, Pentimalli F, Baldassarre G, et al. Transgenic mice overexpressing the wild-type form of the HMGA1 gene develop mixed growth hormone/prolactin cell pituitary adenomas and natural killer cell lymphomas. Oncogene 2005;24:3427-3435.

26 Liau SS, Rocha F, Matros E, Redston M, Whang E. High mobility group AT-hook 1 (HMGA1) is an independent prognostic factor and novel therapeutic target in pancreatic adenocarcinoma. Cancer 2008;113: 302-314.

27 Reeves R, Edberg DD, Li Y. Architectural transcription factor HMGI(Y) promotes tumor progression and mesenchymal transition of human epithelial cells. Mol Cell Biol 2001;21:575-594.

28 Hristov AC, Cope L, Reyes MD, et al. HMGA2 protein expression correlates with lymph node metastasis and increased tumor grade in pancreatic ductal adenocarcinoma. Mod Pathol 2009;22:43-49.

29 Yunis AA, Arimura GK, Russin DJ. Human pancreatic carcinoma (MIA PaCa-2) in continuous culture: sensitivity to asparaginase. Int J Cancer 1977;19:128-135.

30 Jaffee EM, Schutte M, Gossett J, et al. Development and characterization of a cytokine-secreting pancreatic adenocarcinoma vaccine from primary tumors for use in clinical trials. Cancer J Sci Am 1998;4:194-203.

31 Berman DM, Karhadkar SS, Maitra A, et al. Widespread requirement for Hedgehog ligand stimulation in growth of digestive tract tumours. Nature 2003;425: 846-851.

32 Greene FL, Page DL, Fleming ID, et al. . eds. AJCC Staging Manual 6th edn. New York: Springer-Verlag, 2002.

33 Hustinx SR, Leoni LM, Yeo CJ, et al. Concordant loss of MTAP and p16/CDKN2A expression in pancreatic intraepithelial neoplasia: evidence of homozygous deletion in a noninvasive precursor lesion. Mod Pathol 2005;18:959-963.

34 Trapasso F, Sarti M, Cesari R, et al. Therapy of human pancreatic carcinoma based on suppression of HMGA1 protein synthesis in preclinical models. Cancer Gene Ther 2004;11:633-641.

35 Liau SS, Ashley SW, Whang EE. Lentivirus-mediated RNA interference of HMGA1 promotes chemosensitivity to gemcitabine in pancreatic adenocarcinoma. J Gastrointest Surg 2006;10:1254-1262; discussion 1263.

36 Liau SS, Whang E. HMGA1 is a molecular determinant of chemoresistance to gemcitabine in pancreatic adenocarcinoma. Clin Cancer Res 2008;14:1470-1477. 